Rev. Elev. Méd. vét. Pays trop., 1979, 32 (2) : 191-198

\title{
La chèvre et l'utilisation des ressources dans les milieux difficiles
}

\author{
par A. BOURBOUZE et F. GUESSOUS
}

\begin{abstract}
RÉSUMÉ
Après avoir décrit le comportement alimentaire de la chèvre sur les parcours des milieux difficiles, les auteurs étudient son impact sur la végétation naturelle et nuancent les jugements trop sévères dont elle est l'objet. Ils évoquent ensuite les possibilités d'améliorer le système alimentaire en précisant le rôle tenu par les ressources de l'exploitation agricole et concluent en posant le problème de l'intégration de la production caprine dans l'utilisation des ressources forestières et de leur conservation. La fragilité des écosystèmes en place conduit à éviter toute réforme trop brutale ou rapide des systèrnes de production actuellement en vigueur.
\end{abstract}

L'élevage caprin revêt sur le pourtour méditerranéen une importance particulière car il permet la valorisation de surfaces à faible productivité. Fortement contesté, il a fait l'objet d'une polémique qui n'a plus sa place à la lumière des expérimentations entreprises dans divers pays.

Mais les jugements sans appel sur le caprin et son comportement en parcours forestiers se sont traduits dans la plupart des cas par de sévères législations qui hésitent entre l'interdiction pure et simple et le contrôle des effectifs autorisés.

L'analyse des ressources alimentaires de la chèvre en milieu difficile doit s'appuyer sur le fait que les systẻmes de production s'articulent en général sur le parcours d'une part et l'explo1tation agricole d'autre part. Comment l'éleveur combine-t-il ces deux ensembles très contrastés et cependant complémentaires ? Estce que ces deux sous-systèmes s'intervalorisent ?

\section{L'EXPLOITATION DES RESSOURCES PASTORALES PAR LA CHĚVRE}

La chèvre exploite le parcours d'une manière très spécifique. Nous analyserons successivement son comportement alimentaire, la valeur nutritive de sa ration et l'effet de son pâturage sur la végétation.

\section{A. LE COMPORTEMENT ALIMENTAIRE DE LA CHËVRE}

Le comportement alimentaire de la chèvre présente beaucoup d'aspects originaux mais susceptibles de varier fortement selon un certain nombre de facteurs.

\section{Les facteurs liés à l'animal}

La chèvre sélectionne moins parmi les plantes disponibles que le mouton (21). Elle consomme de ce fait une plus grande variété de plantes. Un rapport allemand cité par FRENCH (14) mentionne que la chèvre consomme 449 plantes sur 576 (78 p. 100). EDWARDS (7) souligne qu'en Tanzanie et parmi 67 espèces d'arbres et arbustes, 60 p. 100 sont très appétents. En outre, grâce à ses lèvres très mobiles, sa langue très préhensile, son agilité et sa hardiesse, la chèvre peut accéder à des endroits inaccessibles à d'autres espèces et prélever de la végétation jusqu'à $2 \mathrm{~m}$ de hauteur (22) ou plus si les arbres s'y prêtent (génévrier thurifère, arganier...)

\section{Les facteurs liés à l'aliment}

Dans des formations pastorales où les strates herbacée, chamaephytique, buissonnante et arbustive sont représentées, tous les animaux sélectionnent leur nourriture et la chèvre ne fait pas exception. 


\subsection{Le type de végétation}

La chèvre se porte plus volontiers vers les buissons et arbustes (10). Ainsi, WILSON (29) ventile les coups de dents recensés en 59 p. 100 pour 15 espèces d'arbres et buissons, 33 p. 100 pour 8 espèces de graminées et 8 p. 100 pour 5 espèces diverses. CARRERA (3) sur un relevé de 1721 « coups de dents » calcule que 17 p. 100 seulement de ceux-ci concernent des graminées et 83 p. 100 des arbustes et autres herbacées.

Ces données sont en partie en contradiction avec d'autres résultats plus récents. MALECHEK (23) constate que sur une grande partie de l'année, les chèvres doivent être plutôt considérées comme des " grazers " (consommateurs de graminées) que des «browsers » (consommateurs de buissons). HUSS (19) en conclusion d'une revue, insiste sur le fait qu'il est faux de croire que les arbustes en eux-mêmes sont nécessaires à la ration des caprins.

Il apparaît donc que la chèvre est capable de consommer la végétation ligneuse des arbustes et des buissons, mais qu'elle peut se porter également plus volontiers vers la strate herbacée.

\subsection{Le stade de développement de la végétation}

Pour mieux expliquer les choix opérés par le caprin, les observations doivent tenir compte des différents stades de développement de la végétation intertype et intratype.

a) Différences entre types de végétation

De nombreux auteurs ont remarqué que les buissons sont appétés en saison sèche et les graminées et autres herbacées en saison humide $(27,7,13)$. Au Texas, les arbustes constituent plus de 50 p. 100 de la ration de juillet à août et de novembre à février (13). MALECHEK (24) au moyen de fistules cesophagiennes, montre que les graminées, malgré la disponibilité en arbustes, sont prépondérantes dans la ration de juin à octobre et que ces mêmes arbustes sont consommés en toute saison, sans relation étroite avec leur disponibilité (fig. 1). Il apparaît donc que la disponibilité d'un type de végétation d'une saison à l'autre n'est pas forcément un bon indicateur de son utilisation et que le stade de développement de cette végétation entre en ligne de compte.

Dans le Sud tunisien, les chèvres ne consomment les plantes herbacées annuelles qu'au printemps et passent rapidement sur les pérennes de la strate chamaephytique le restant de l'année. En cas de fortes pluies, le passage des annuelles aux pérennes s'effectue plus tard en saison.

b) Différences au sein d'un même type de végétation

Pour un même type de végétation, l'effet saisonnier est également manifeste. Sur des parcours texans par exemple, les arbustes sont consommés dans un ordre bien déterminé selon leur degré d'acceptabilité ; en janvier le chêne à feuilles pérennes, en février le génévrier car le chêne quoique toujours vert se dépouille d'une partie de ses feuilles; de mars jusqu'à décembre, seul le chêne à feuilles caduques dans sa phase végétative est consommé (23).

FIGURE 1

\section{POURCENTAGE DES DIFFÉRENTS TYPES DE VÉGÉTATION DANS LA RATION DE CHÈVRES}

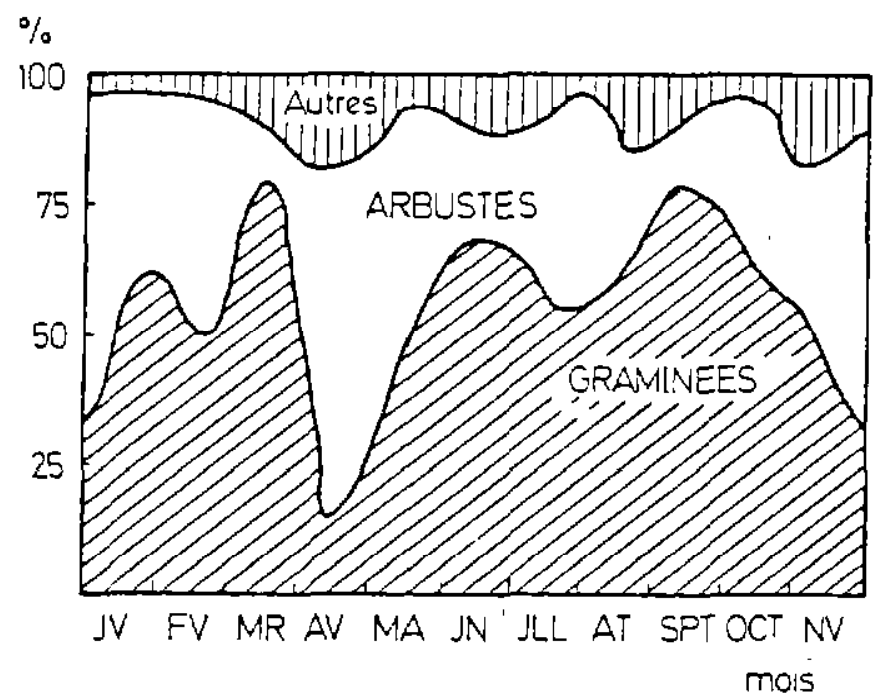




\subsection{L'appétence relative des constituants d'une même espèce}

Chez les graminées, les inflorescences sont surtout prélevées. Sur les arbustes, la chèvre préfère les feuilles aux tiges et aux fruits (23). Dans certains cas, seuls les rejets ou les jeunes feuilles dépassant $60 \mathrm{~cm}$ de hauteur sont consommés (29).

\subsection{Accessibilité des parties appétentes}

C'est un facteur qui, avec raison, est tenu pour très important $(30)$.

Il apparaît ainsi clairement que la chèvre sélectionne sa ration beaucoup plus en fonction du stade de croissance des plantes que de l'espèce proprement dite $(29,18)$.

\section{Facteurs liés à la conduite du troupeau : la charge à l'hectare}

Les expérimentations et observations qui ont porté sur ce facteur de variation du comportement alimentaire sont rares. MALECHEK (23) indique que globalement la proportion d'arbustes, de graminées et d'autres herbacées consommés change peu d'une charge forte (1 caprin/5 ha) à une charge faible (1 caprin/16 ha), mais que l'effet saison est très net ; en début de printemps, arbustes et graminées sont consommés sous charge forte contre graminées et autres herbacées sous charge faible.

\section{B. COMPORTEMENT COMPARÉ DE LA CHËVRE EN TROUPEAUX PLURISPÉ- CIFIQUES}

Au Maghreb, le système d'exploitation repose souvent sur l'utilisation des caprins et des ovins rassemblés dans un même troupeau, ce qui peut présenter des avantages compte tenu de leurs comportements alimentaires différents $(27,25$, 19).

En effet CORY en 1927 (5) avait déjà relevé ces différences pour quelques ruminants conduits sur un même parcours (tabl. I).

TABL. $\mathbb{N}^{\circ} I$-Répartıtıon du temps de prise alımertaire entre types de végétation pour différentes espèces animales (0.100) (Cory, 1927)

\begin{tabular}{|c|c|c|c|}
\hline Types de végétation & Bovins & Ovins & $\begin{array}{l}\text { Chèvre } \\
\text { Angora }\end{array}$ \\
\hline Pâturage d'herbacëes & 76,0 & 79,0 & 38,1 \\
\hline Broutage d'arbustes & 8,5 & 10,1 & 53,1 \\
\hline Divers et suppléments & 15,5 & 10,0 & 8,8 \\
\hline
\end{tabular}

TABL. $\mathbb{N}^{\circ} I I-I m p o r t a n c e$ de la strate arbustive dans la ration d'ovins et caprins assocıês sur parcours. (wils on 1972)

\begin{tabular}{|c|c|c|c|}
\hline $\begin{array}{c}\text { En p.100 du total } \\
\text { consommé }\end{array}$ & Novembre & Fivrice & Juin \\
\hline Caprins & 67 & 78 & 87 \\
\hline Ovins & 21 & 10 & 19 \\
\hline
\end{tabular}

Par ailleurs WILSON (30) note dans un essai où chèvres et moutons pâturent simultanément que les rations sont sensiblement différentes (tabl. II). Il affirme le caractère de complémentarité des deux espèces, malgré la compétition sur les graminées. La productivité optimale supposerait donc le pâturage simultané des ovins et des caprins.

Dans la steppe présaharienne du Sud tunisien, NOVIKOFF et GRIEGO (26) montrent que les chèvres exploitées seules consomment au printemps plus d'annuelles (64 à 75 p. 100 de la ration) qu'associées aux ovins (15 à 30 p. 100). Ultérieurement en saison humide, les caprins consomment plus volontiers les tiges sèches

TABL. $\mathrm{X}^{0}$ III-Variations de la corpcsitiov ce la ration des ovins et des caprins sur un parcours forestier. (Belrbouze, Derkaoui 1977). (exprimëe en p.100 des quartités ingérées).

\begin{tabular}{|c|c|c|c|c|c|}
\hline \multirow{2}{*}{ 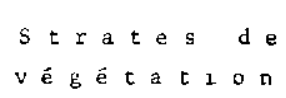 } & \multicolumn{3}{|c|}{$\mathrm{C}^{\prime} \mathrm{a} \mathrm{p} \times \mathrm{i} \mathrm{s}$} & \multicolumn{2}{|c|}{$a v 1 n s$} \\
\hline & Déçembre & Fëvrier & Avril & Fêvrier & Avril \\
\hline Arb orêe & 27,4 & 29,5 & 9,2 & 5,5 & 1 \\
\hline Arbustive & 5,4 & 16,6 & 3,9 & 2,0 & 0 \\
\hline $\begin{array}{l}\text { Chamaephytique et } \\
\text { buissonnante }\end{array}$ & $2 B, 7$ & 18,6 & 31,0 & 2,0 & 3,5 \\
\hline Herbacëe & 38,5 & 35,3 & 55,9 & 90,5 & 93,5 \\
\hline (Graminées) & $(6,7)$ & $(9,5)$ & $(18,2)$ & $(46,0)$ & $(47,0)$ \\
\hline (Autres herbacēes) & $(31,8)$ & $(25,8)$ & $(37,7)$ & $(44,5)$ & $(46,5)$ \\
\hline
\end{tabular}


dressées et les ovins la litière sèche. Au total, sur l'ensemble de l'année, la compétition entre les deux espèces animales porte sur peu de plantes et pendant peu de temps.

Un autre travail sur des parcours forestiers montagnards du semi-aride froid du Haut Atlas marocain (1) s'est attaché à définiı les niches alimentaires des ovins et caprins à différentes saisons afin de juger de la place particulière des caprins dans cet écosystème forestier (tabl. III).

Il apparaît que la strate arborée (chêne vert et génévrier) exploitée par le forestier ne participe pas de façon très importante au régime de la chèvre. La compétition entre ovins et caprins porte plus sur les non-graminées que sur les graminées elles-mêmes.

\section{VALEUR NUTRITIVE DES RATIONS}

Bien que les avis ne concordent pas toujours, il semble bien que la chèvre ne digère pas mieux les aliments que les autres espèces animales. La difficulté de faire des comparaisons expérimentales sans reproches est grande car les comportements alimentaires, les rythmes de consommation, les stades physiologiques sont souvent différents d'une espèce à l'autre. Nous ne reviendrons pas sur cette controverse alimentée par de nombreux auteurs : $(6,20,8,15,16 \ldots)$.

\section{L'IMPACT DE LA CHEVRE SUR LA VEGETATION}

Pour mesurer l'impact de la chèvre sur la végétation, il est indispensable d’apprécier non seulement la quantité de fourrage prélevée, c'est-à-dire la production pastorale, mais aussi les tendances évolutives du parcours. Or peu d'auteurs l'ont fait de façon précise car les protocoles correspondants sont lourds et doivent s'étendre sur plusieurs années. Rares sont donc les résultats qui dépassent la simple observation dans ce domaine.

Il y a longtemps déjà que HORNBY (17) a souligné que la chèvre, mieux adaptée à des conditions précaires, est seule présente dans les derniers stades de dégradation d'un parcours à laquelle ont largement participé bovins et ovins. En réalité, elle ne fait que compléter la destruction en broutant les strates buissonnantes et arborées qui persistent en dernier lieu. Seule en cause au stade ultime; elle est globalement jugée responsable. En fait, ce qui est en cause, c'est tout ur système d'exploitation inadapté, caractérisé par un surpâturage incontrôlé et continu.

D'après HUSS, la plupart des dégradations pastorales à travers le monde sont dues au surpâturage par les ovins et les bovins dont l'agressivité envers la strate herbacée et envers le sol par piétinement déclenche en premier lieu le processus d'érosion. Ajoutons que le rôle du berger est décisif : dans les parcours forestiers du Haut Atlas Central (2), la part des feuillages dans la ration hivernale des ovins et des bovins dépasse 30 p. 100 si l'on comptabilise simplement ce qui est coupé par le berger (génévrier pour ovins et caprins, chêne vert pour les bovins). Cependant la chèvre est responsable de dégâts directs dans certaines conditions d'exploitation. MERRIL (26) montre que de jeunes arbustes se développent mal avec une charge par ha légère en caprins, par contre, leur développement s'effectue correctement en présence d'ovins et de bovins. Par ailleurs, le broutage excessif du feuillage entraîne une baisse de la production (4). FRENCH (14) à la suite de beaucoup d'autres, conclut que la chèvre, de par son comportement alimentaire spécifique, favorise la croissance anormale des jeunes plantations.

De nombreuses autres publications mettent cependant en relief les avantages que l'on peut tirer de l'utilisation des caprins sur certains types de végétation. En Tanzanie, STAPLES (28) sur des parcours débroussaillés puis réensemencés en Cynodon, compare le pâturage de 2 bovins et celui de 14 caprins par parcelle sur une période de 4 ans. Au terme de l'essai, la parcelle réservée aux bovins est réembroussaillée et la végétation herbacée est faible ; celle réservée aux caprins comprend une bonne végétation herbacée mais beaucoup de mauvaises herbes.

MAGEE (1957) sur 15 ranches texans observe que la chèvre utilisée pour contrôler les repousses après défriche, paye par ses produits le coût du défrichement en empêchant ou en retardant le réembroussaillement. WILSON (30) souligne que le contrôle de l'embroussaillement ne peut être réalisé qu'avec des charges à l'hectare trop fortes, incompatibles avec une bonne productivité du troupeau.

Ainsi, tous les animaux peuvent être jugés agressifs si les modes d'exploitation des parcours ne sont pas adaptés aux habitudes alimentaires du bétail utilisé.

Un premier bilan de l'utilisation du parcours par les caprins permet donc d'affirmer que ce troupeau, par l'originalité de son comportement 
FIGURE 2

BILAN ALIMENTAIRE DES CAPRINS

(Bourbouze 1976)

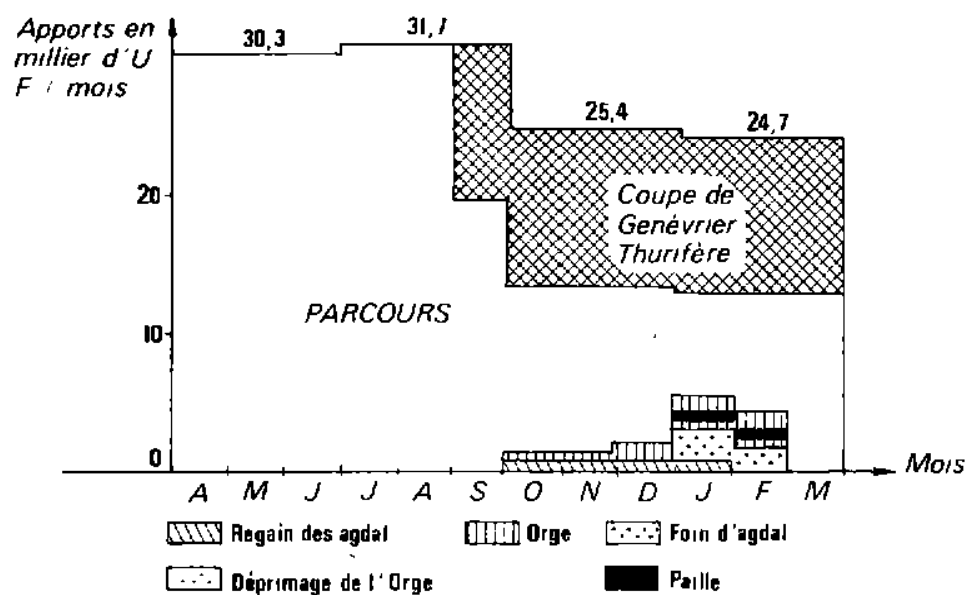

alimentaire et par la spécificité de son système de production, occupe une position tout à fait particulière et irremplaçable.

Les ressources dont ce troupeau dispose, ne proviennent cependant pas toutes du parcours. L'exploitation agricole proprement dite joue un rôle, qu'on pense souvent à tort négligeable.

\section{LES RESSOURCES ALIMENTAIRES DE LA CHËVRE DANS L'EXPLOITATION AGRICOLE}

On sait l'exiguité des exploitations agricoles des zones difficiles où la chèvre est présente. Les ressources alimentaires tirées de celles-ci sont pourtant très variées. En effet, toutes les sources possibles sont exploitées. Par ordre d'importance, on peut classer les aliments utilisés selon leur origine :

- en premier lieu on trouve les aliments concentrés produits et/ou achetés, tels que les céréales ;

- puis les sous-produits des cultures et les fourrages de " récupération " dont le coût n'est pas calculable (paille et adventices en prove- nance du désherbage manuel des céréales, cueillette dans les bords de chemins ou de seguias...) ;

- enfin sur des soles spécifiques, le fourrage produit est, en certaines circonstances très rares, réservé aux caprins.

Aussi dans un système pastoral, s'il est vrai que le caprin ne reçoit que fort peu d'aliments en provenance de l'exploitation, le peu qu'il reçoit est distribué dans des périodes stratégiques du cycle productif de l'animal : début de lactation des mères en hiver, alimentation du chevreau de 0 à 2 mois.

Sur les steppes de l'Oriental marocain, les caprins tirent 97 p. 100 de leurs besoins du parcours en année moyenne, et 76 p. 100 en année mauvaise (9). D'après la même étude, sur $100 \mathrm{UF}$ d'alimentation complémentaire, 83 UF sont de lorge, $15 \mathrm{UF}$ du foin de luzerne et $2 \mathrm{UF}$ de la paille.

Dans le Haut Atlas Central marocain, 94 p. 100 des ressources proviennent des parcours sur l'ensemble de l'année contre 77 p. 100 uniquement en janvier (2) (fig. 2). La répartition des ressources alimentaires entre les différentes

TABL. N'IV-Répartıtıon entre espèces de ressources alimentaires en provenance de l'exploitation agricole et des parcours. (Bourbouze 1976).

\begin{tabular}{|c|c|c|c|c|c|}
\hline Provenance & Caprins & Ovins & Bovins & Equzdés & \\
\hline $\begin{array}{c}\text { Exploitation } \\
\text { agricole }\end{array}$ & 12.100 & 8 p. 100 & $56 \mathrm{p} .100$ & 24 p. 100 & 10) p. 100 \\
\hline Parcours & 49 p. 100 & -32 - 300 & $1 \% \mathrm{p} .100$ & $5 \mathrm{p} .100$ & $100 \Gamma .100$ \\
\hline
\end{tabular}


espèces animales exploitées dans cette région souligne mieux la place tenue par la chèvre dans ce système (tabl. IV).

Dans ces conditions, l'exploitation agricole peut-elle participer plus à l'alimentation des caprins ?

- Les céréales étant commercialisées à des prix très élevés, une chèvre dont la productivité ne dépasse pas $10 \mathrm{~kg}$ de croît par an n'arrive pas à valoriser suffisamment les unités fourragères consommées. De ce fait, l'éleveur est condamné à une extrême parcimonie et la complémentation ne saurait dépasser 20 p. 100 du total des unités fourragères par an.

- Les exploitations sont de taille très réduite et la sole céréalière est prépondérante pour couvrir les besoins d'autoconsommation. Corrélativement, la place des fourrages cultivés est très minime. Les réserves stockées pour le troupeau ovin-caprin ne permettent pas une survie de plus de 20 jours environ.

Par ailleurs, dans les zones où les variations climatiques interannuelles sont fortes, produire son fourrage n'est pas toujours ressenti comme une nécessité par l'éleveur car d'autres stratégies sont possibles en année de disette : acheter du fourrage ou du concentré, louer des chaumes, accepter l'amaigrissement des animaux, vendre une partie de ses animaux, donner les animaux en association...

Selon la conjoncture l'une ou l'autre de ces solutions est adoptée. C'est l'impossibilité de prévoir la quantité de fourrage nécessaire qui limite aussi l'extension de la sole' fourragère dans les milieux difficiles aux fortes variations climatiques. Avec des troupeaux infiniment plus importants, le "manager" australien tient des raisonnements de ce type.

\section{LES VOIES DE RECHERCHES POUR L'AMÉLIORATION DES SYSTÊMES ALIMENTAIRES DES CAPRINS}

L'étroite dépendance des aspects techniques et socio-économiques a toujours retardé l'amélioration de ces systèmes de production. Ces contraintes seront rapidement citées avant d'évoquer des voies de recherche possibles.

\section{A. LES CONTRAINTES SOCIO-AGRONO- MIQUES}

1) La première contrainte qui entrave le développement de la production pastorale est liée à l'exiguïté des exploitations agricoles qui font partie de ce système. Et il n'est pas possible de porter remède au déficit alimentaire du troupeau caprin durant la période de repos végétatif des parcours, en faisant appel à une restructuration de l'exploitation agricole. Les seules actions envisageables concernent par conséquent l'amélioration pastorale proprement dite et le recours à des ressources extérieures à la zone pour constituer des réserves alimentaires. La dépendance du caprin vis-à-vis des ressources pastorales est inhérente au système et ne peut être évitée.

2) Le statut des parcours est aussi une puissante entrave au développement.

L'explosion démographique des dernières décennies s'est accompagnée de la mise en culture d'une partie des parcours collectifs. L'ancien système pastoral a fait place à un mode d'exploitation défaillant où l'égalité théorique des droits de pâturage n'est plus respectée.

Le statut domanial est souvent contesté et la réglementation des usages en forêt est inadaptée. Les autorités administratives confrontées à une forte résistance des éleveurs et mieux informées de l'importance économique du troupeau caprin, considèrent maintenant le problème de l'élevage caprin de manière plus positive et si les réglementations ne sont pas toujours modifiées, dans bien des cas elles sont peu appliquées.

\section{B. QUELQUES VOIES DE RECHERCHE POUR L'AMÉLIORATION DES SYS- TEMES ALIMENTAIRES DES CA- PRINS EN MILIEU DIFFICILE}

L'objectif est d'évaluer la quantité de végétation prélevable, en forêt ou hors forêt, sans préjudice pour l'équilibre de l'écosystème.

Dans un récent rapport sur les relations entre l'animal et le pâturage en zone forestière, FLAMAND (12) insiste justement sur le rendement optimal de l'écosystème qui se pose en termes d'équilibre animal-forêt. Quel est le point d'équilibre entre l'animal et la forêt ? Comment un système de production caprine peut-il intégrer l'utilisation de la forêt ?

Deux voies de prospection peuvent s'ouvrir. La première est d'ordre historique et consiste à enquêter sur les modes de production de ce type, tombés souvent en désuétude. C'est le cas par exemple de ZIANI (31) qui relève fidèlement le système de production très complexe des chèvres de Jegar en Dalmátie. 
La deuxième voie de prospection, plus familière, est d'ordre expérimental. Les principales pistes de travail, relevées déjà par FLAMAND (12) concernent :

- L'étude des dommages causés à la végétation - aux arbres en particulier - par la chèvre. L'existence, l'importance et la nature des dommages dépendent du type de végétation, de la charge de bétail à l'hectare et de la période de pâturage ;

- Les effets sur les sols : effets physiques (tassement) et chimiques ;

- Les recherches sur l'utilisation des caprins comme agent de gestion du matorral ou de la forêt : possibilités de débroussaillement, valorisation de l'engrais mis à la plantation, protection contre les incendies...;

- Le point d'équilibre entre chèvre et forêt : possibilités de prélèvement sur la biomasse disponible en relation avec la production de bois, densité optimale du peuplement forestier :
- L'effet du troupeau plurispécifique sur les parcours ;

- L'effet sur la végétation selon les types caprins. Le croisement avec des races européennes modifie-t-il le comportement alimentaire du caprin des milieux difficiles ?

- L'intégration du parcours dans le cycle de production animale avec analyse de la place de l'exploitation agricole dans ce système, les possibilités de constitution de réserves alimentaires, le stockage de la ramée;

- La réponse de la chèvre à l'aménagement du parcours ; étude des effets des mises en défens de longue durée ou temporaires, de la rotation, conséquence des actions directes sur le sol et la végétation.

C'est donc un travail considérable qui reste à accomplir avant de vouloir réformer trop brutalement les systèmes de production en place.

\title{
SUMMARY
}

\section{Goat and resource utilization in precarious environments}

\begin{abstract}
After the description of the alimentary behaviour of the goat in rangelands of precarious environments, the authors study its impact on natural vegetation and conclude that it has been overrated. They review the nutrition improvement possibilities taking into account the agricultural outputs and rise the problem of the integration of the goat production into the utilization and preservation of forest resources. The fragility of the present ecosystems leads to avoid every drastic or fast reform of the current production systems.
\end{abstract}

\section{RESUMEN}

La cabra y la utilización de los recursos en los medios dificiles

Después de una descripción del comportamento alimenticio de la cabra sobre los pasturajes de los medios dificiles, los autores describen su impacto sobre la vegetación natural y matizan los juicios demasiado severos de que son objeto. Luego tratan las posibulidades de mejorar el sistema alimenticio al especificar el papel desempeñado por los recursos de la explotación agricola y concluyen al plantear el problema de la integración de la producción cabruna en la utilización de los recursos forestales y de su conservación. La fragilidad de los ecosistemas existentes hace evitar toda reforma demasiado brutal o rápida de los sistemas de producción actualmente en vigor.

\section{BIBLIOGRAPHIE}

1. BOURBOUZE (A.), DERKAOUI (M.). Comportements alimentaires comparés des ovins et caprins en troupeau commun sur des parcours forestiers de montagne. Rabat, Institut Agronomique et Vétérinaire Hassan II, 1977.

2. BOURBOUZE (A.), DONADIEU (P.) et HAMMOUDI (A.). L'unité montagnarde de développement intégré de la vallée de l'Azzaden du Haut Atlas Central. Rabat, I. A.V. H., 1976, II.
3. CARRERA (C.). Tipos de plantes que consume el ganado caprino. Mexico, ITESM, Monterrey, N. L., 1971, p. 168-169.

4. COOK (C.), KOTHMANN (M.), HARRIS (L. E.). Effect of range conditions and utilization on nutritive intake of sheep on summer ranges. $J$. Range $\mathrm{Mgmt}$, $1965,18: 69-73$.

5. CORY (V. L.). Activities of livestock on the range. Texas Agric. exp. Sin Bull., 1927: 367. 
6. DEVENDRA (C.), BURNS (M.). Goat production in the tropics. Bucks, G. B., C. A. B., 1970, 177 p.

7. EDWARDS (C. C.). Some notes on the food of goats in a semi-arid area. E. Afr. agric. J., 1948, $13: 221$.

8. EL HAG. A comparative study between desert goats and sheep efficiency of food utilization. WId Rev. anim. Prod., 1976, 12 : 43-48.

9. E. R. E. S. Etude pour l'aménagement des terrains de parcours du Maroc Oriental. Rabat, DMV-Mara, 1972, Fasc. 31.

10. F. A. O. Pâturage et forêt. Rome, F. A. O., 1952. (Etude $n^{0} 27$. )

11. F. A. O. Séminaire sur les politiques d'élevage des chèvres dans le bassin méditerranéen et le ProcheOrient. Athènes, 1964. Rome, F. A. O., 1965, п ${ }^{\circ} 1929$.

12. FLAMAND (J. C.). L'animal et le pâturage en zone forestière et marginale. Conférence scientifique M. A. B., Montpellier, Oct. 1976, 24 p.

13. FRAPS (G. S.), CORY (V. L.). Composition and utilisation of range vegetation of sutton and Edwards comities. Tex. Agric. exp. Stn. Bull., 1940, 586, 39 p.

14. FRENCH (M. H.). . Obseryation sur la chèvre. Rome, F. A. O., 1971. (Etudes agricoles de la F. A. O., $\mathrm{n}^{\circ}$ 80.)

15. GIHAD (E. A.). Intake, digestibility and nitrogen utilization of tropical natural grass hay by goats and sheep. J. anim. Sci., 1976, 43 (4) : 879-883.

16. GRIEGO (R. R.). Spring grazing and bioenergeties studies of sheep and goats. Logan (Utah), Desert BIOME, 1974. (Progress report no 3.)

17. HORNBY (H. E.). Overstocking in Tanganika Territory. E. Afr. Agric. J., 1936, $1: 353-360$.

18. HUSS (D. L.), ZERTUCHE (J. M.). Informe sobre el proyecto de experimentación para el aprovechamiento con ganado caprino con vegetación tipo matorral. Monterrey, Mexico, ITESM, 1970, 48 p.

19. HUSS (D. L.). Goat response to use of shrubs as forage (review). Int. Symposium. Logan (Utah) juillet 1971.
20. JANG (S.). MAJUMDAR (B. N.). A study of comparative digestibilities in different species of ruminants. Ann. bioch. exp. Med., 1962, 22: 303-308,

21. LE HOUEROU (H. N.). Séminaire F. A. O. sur les politıques d'élevage des chèvres dans le bassin méditerranéen et le Proche-orient, Athènes, 1964.

22. MAHER. The goat : friend or foe ? E. Afr. agric. $J$, 1945.

23. MALECHEK (J. C.), LEIN WEBER (C. L.). Forage selectivity by goats on lightly and heavily grazed ranges. Range Mgmt, 1972 (2) V51 95 : 105-111.

24. MALECHEK (J. C.), LEIN WEBER (C. L.). Chemical composition and in vitro digestibility of forage consumed by goat on lightly and heavily stocked ranges : J. anim. Sci., 1972, 35 (5) : 1014-1019.

25. MERRIL (L. B.). Results of grazing single classes of Livestock in combination with several classes. Tex. Agric. exp. Stn., 1954 (1726), 7 p.

26. NOVIKOFF (G.), GRIEGO (R.). US/IBP Desert BIOME. Séminaire arabe sur les parcours, Rabat, mars 1977.

27. PALAZON (J.). Ganado cabrio. Salvat. Ed., 1953, $487 \mathrm{p}$.

28. STAPLES (R. R.), HORNBY (R. M.). A study of the comparative effects of goats and cattle on a mixed grass bush pasture; E. Aft. agric. J., 1942 : 62-70.

29. WILSON (P. M.). A review of browse in the nutrition of grazing animals. J. Range Mgmt., 1969, 22 (1) : 25-28.

30. WILSON (P. M.). Comparison of the diets of goats and sheep on a woodland community in western New South Wales (Australia). Austr. J. exp. Agric. anim. Husb., 1972, 15 : 45-53.

31. ZIANI (P.). Les perspectives et les principes de la technique de l'élevage des chèvres dans les pays méditerranéens. F. A. O./Sem. FOR (Chèvre)/11, 1964 